\title{
Social Motor Priming: When offline interference facilitates motor execution
}

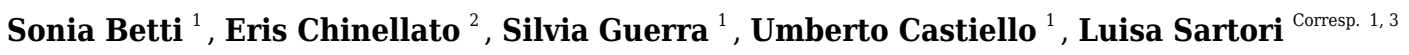 \\ 1 Dipartimento di Psicologia Generale, Università degli Studi di Padova, Padova, Italy \\ 2 Department of Design Engineering and Mathematics, Middlesex University, London, United Kingdom \\ 3 Padova Neuroscience Center, Università degli Studi di Padova, Padova, Italy \\ Corresponding Author: Luisa Sartori \\ Email address: luisa.sartori@unipd.it
}

Many daily activities involve synchronizing with other people's actions. Previous literature has revealed that a slowdown of performance occurs whenever the action to be carried out is different to the one observed (i.e., visuomotor interference). However, action execution can be facilitated by observing a different action if it calls for an interactive gesture (i.e., social motor priming). The aim of this study is to investigate the costs and benefits of spontaneously processing a social response and then executing the same or a different action. Participants performed two different types of grips, which could be either congruent or not with the socially appropriate response and with the observed action. In particular, participants performed a precision grip (PG; thumb-index fingers opposition) or a wholehand grasp (WHG; fingers-palm opposition) after observing videos showing an actor performing a PG and addressing them (interactive condition) or not (non-interactive condition). Crucially, in the interactive condition, the most appropriate response was a WHG, but in 50 percent of trials participants were asked to perform a PG. This procedure allowed us to measure both the facilitator effect of performing an action appropriate to the social context (WHG) - but different with respect to the observed one (PG) - and the cost of inhibiting it. These effects were measured by means of 3-D kinematical analysis of movement. Results show that, in terms of reaction time and movement time, the interactive request facilitated (i.e., speeded) the socially appropriate action (WHG), whereas interfered with (i.e., delayed) a different action (PG), although observed actions were always PG. This interference also manifested with an increase of maximum grip aperture, which seemingly reflects the concurrent representation of the socially appropriate response. Overall, these findings extend previous research by revealing that physically incongruent action representations can be integrated into a single action plan even during an offline task and without any training. 
1 Social Motor Priming: When offline interference facilitates motor execution

3 Sonia Betti ${ }^{1}$, Eris Chinellato ${ }^{2}$, Silvia Guerra ${ }^{1}$, Umberto Castiello $^{1}$ and Luisa Sartori ${ }^{1,3 *}$

4

$5 \quad$ DDipartimento di Psicologia Generale, Università degli Studi di Padova, Padova, Italy.

$6 \quad{ }^{2}$ Department of Design Engineering and Mathematics, Middlesex University, London, United

7 Kingdom.

8 3Padova Neuroscience Center, Università degli Studi di Padova, Padova, Italy.

11 Corresponding author:

12 Luisa Sartori

13 Dipartimento di Psicologia Generale, Università degli Studi di Padova,

14 Via Venezia 8, 35131, Padova, Italy

15 E-mail address: luisa.sartori@,unipd.it 


\section{Abstract}

18 Many daily activities involve synchronizing with other people's actions. Previous literature has revealed that a slowdown of performance occurs whenever the action to be carried out is different to the one observed (i.e., visuomotor interference). However, action execution can be facilitated by observing a different action if it calls for an interactive gesture (i.e., social motor priming). The aim of this study is to investigate the costs and benefits of spontaneously processing a social response and then executing the same or a different action. Participants performed two different types of grips, which could be either congruent or not with the socially appropriate response and with the observed action. In particular, participants performed a precision grip (PG; thumb-index fingers opposition) or a whole-hand grasp (WHG; fingers-palm opposition) after observing videos showing an actor performing a PG and addressing them (interactive condition) or not (non-interactive condition). Crucially, in the interactive condition, the most appropriate response was a WHG, but in 50 percent of trials participants were asked to perform a PG. This procedure allowed us to measure both the facilitator effect of performing an action appropriate to the social context (WHG) - but different with respect to the observed one (PG) - and the cost of inhibiting it. These effects were measured by means of 3-D kinematical analysis of movement. Results show that, in terms of reaction time and movement time, the interactive request facilitated (i.e., speeded) the socially appropriate action (WHG), whereas interfered with (i.e., delayed) a different action (PG), although observed actions were always PG. This interference also manifested with an increase of maximum grip aperture, which seemingly reflects the concurrent representation of the socially appropriate response. Overall, these findings extend previous research by revealing that physically incongruent action representations can be integrated into a single action plan even during an offline task and without any training. 
40

41

42

\section{Introduction}

A wealth of research has shown that motor response is facilitated (e.g., shorter reaction times and increased accuracy) if the action to be carried out has been recently observed. This phenomenon, termed visuomotor priming, has been defined as the facilitation to perform an action congruent with the observed one (Heyes, 2011). Interestingly, visuomotor priming can be reversed when others' actions are instrumental for the fulfillment of a specific joint goal. If, for example, someone holding a mug by its handle - using a three-digit grasp - hands it to us, we automatically grasp the 'available' surface with a whole-hand grip, rather than imitating the observed grip. In this case, the two actions are physically incongruent, yet complementary. Recent findings have shown that the brain can easily resolve the conflict between the automatic tendency to imitate, and the need to perform context-related complementary actions (Sacheli et al., 2015; Era et al., 2018; for a review see Sartori \& Betti, 2015). Brief periods of sensorimotor experience, in which participants are trained to perform a different action from the one observed, can even abolish (Cook et al., 2010) or reverse (Catmur et al., 2008) visuomotor priming. It then appears that action observation does not automatically lead to imitation, but rather, depending on sensorimotor experience, observed actions could prime different responses.

Learnt social responses can modulate motor performance (Liepelt, Prinz \& Brass, 2010;

Flach et al., 2010), so that complementary response preparation can spontaneously overwhelm imitative responses (i.e., social motor priming; Wang \& Hamilton, 2013; Hamilton, 2013).

Liepelt and colleagues (2010) described the reversed compatibility effect when observing a human right hand extended for a handshake: participants responded faster with their own right hand, not with their mirror hand (Liepelt, Prinz \& Brass, 2010). This effect is driven by the strongly life-long learnt social response of responding with the non-mirror hand to handshaking. 
63 Continuing on this analysis, also prosocial words (e.g., "join") shown prior to action observation

64 are able to shape perception and behavior (Leighton et al., 2010). Of relevance for the present

65 study, verbal social primes can increase interference when participants execute an action

66 different from the observed one (Sparks et al., 2016). Along with visuomotor effects, visuomotor

67 interference - a higher variance on action execution when participants observe incompatible vs

68 compatible movements - has been described (Gandolfo et al., 2019; Kilner et al., 2003). To date,

69 only few studies have considered how visuomotor interference is modulated by social contexts.

We recently provided evidence that online interference occurs when an observed movement

requires an incongruent grasp with respect to the prehension simultaneously observed and

executed (Chinellato, Castiello \& Sartori, 2015). This result suggests that observing an

interactive gesture automatically generates an internal representation of the most appropriate

response. Such representation can cause interference in the simultaneous execution of a different

grasping movement, due to competition between the two motor plans (i.e., online interference).

Here, we aim to extend previous results by investigating whether visuomotor interference can

modulate perception-action coupling even after action observation (i.e., offline interference).

So far, most of the studies investigating social interactions have been confined to settings

where participants were instructed (or trained) to perform similar (imitative blocks) or dissimilar (complementary blocks) actions (Ocampo \& Kritikos 2010; Ocampo, Painter \& Kritikos, 2012;

Newman-Norlund et al., 2007; Poljac, van Schie \& Bekkering, 2009; van Schie, van

Waterschoot \& Bekkering, 2008). Here, the paradigm did not entail any imitative or complementary blocks and no instructions were given before the trials. An auditory Go signal was released just after video presentation, signaling which type of response had to be executed.

Notably, this procedure let participants prepare a natural motor response, that could be 
86 incidentally congruent or incongruent with respect to the subsequent requested gesture. When

87 participants are instructed to actively oppose an observed action (e.g., during complementary

88 blocks), conflict detection and response selection processes are engaged in advance of viewing

89 the action rather than in reaction to it (Cross \& Iacoboni, 2014a). As compared to incidentally

90 matched or mismatched stimulus-response pairs, intentional imitation or counter-imitation

91 widely recruit top-down cognitive control networks (Campbell \& Cunnington, 2017) and might

92 suppress motor resonance (Bardi et al., 2015; Cross \& Iacoboni, 2014b).

93

In order to induce social motor priming, we capitalize here on a well-established reach-to-

94 grasp paradigm (for a review, see Sartori \& Betti, 2015). Kinematic analysis of reach-to-grasp

tasks is more effective in detecting subtle effects in social contexts, compared to simple reaction time measurements used in classic intransitive tasks (e.g., tapping) (for a review, see KrishnanBarman, Forbes \& Hamilton, 2017). To induce a full range of facilitation/interference effects, we devised a full-factorial experimental design (Fig. 1). Participants observed two videos of an actress: i) grasping a tablespoon with a precision grip (PG), pouring sugar in a mug located nearby, and then stretching out her arm trying to pour some remaining sugar into a mug located out of her reach, in the observer's direction (Interactive action; Fig. 2a), ii) grasping a tablespoon with a PG, pouring sugar into the same first mug, and then returning to the starting point (NonInteractive action; Fig. 2b). Notably, a number of studies has shown that this kind of interactive requests spontaneously elicits a complementary response in the observers (i.e., social affordances; e.g., Scorolli et al., 2014; for a review see Sartori \& Betti, 2015; Sartori 2016). Here, viewing the actress stretching out her arm without being able to pour sugar in the mug

107 located in the video foreground - therefore closer to the participant, triggered in the participant 108 the socially appropriate response (i.e., a whole-hand grasp -WHG- toward the mug, to grasp it 
109 and bring it closer to the actress). Participants had to observe these perceptual events, to wait for

110 an auditory 'Go' signal, and then to either grasp a spoon with a PG (50\% of trials) or a mug with

111 a WHG (50\% of trials), depending on the 'Go' signal. Two baseline conditions, in which

112 participants simply observed a fixation cross and performed the grasping tasks (i.e., PG and

113 WHG trials), were also set. Given that participants always observed a PG action performed by

114 the actress (i.e., grasping the spoon), we would expect a facilitation effect when they too grasped

115 the spoon (visuomotor priming) to the detriment of performing a different action (visuomotor

116 interference). On the other hand, if the social request elicits the preparation of a WHG in

117 response to what is observed, in the Interactive condition we should expect a facilitation when

118 performing a WHG (social motor priming) with respect to a PG (social motor interference). See

119 Figure 1 for a schematic representation of this set of hypotheses. With this in mind, the aim of

120 the present study was to specifically investigate the benefits and costs associated with the

121 processing of a social request in a postponed task. In particular, we tested whether offline social

122 interference only affects reaching parameters or it influences kinematics at all levels (i.e., both

123 the reaching and grasping components). Notably, the output of the present paradigm would differ

124 from the classic Stimulus-Response Compatibility effect (Fitts \& Seeger, 1953) in which

125 participants are instructed to perform a finger tapping in response to observing a finger tapping

126 (compatible) or a finger lifting (incompatible). Here, no instruction was given before the trial in

127 terms of motor preparation, and the observed grasp was always a PG.

129 Materials \& Methods

130

Participants 
131 Sixteen right-handed volunteers (10 females and 6 males, between the ages of 21 and 31)

132 participated in the experiment. A right-handed non-professional actor (female, 28 years old) was

133 recruited for video-clips recording. All participants gave their informed written consent to

134 participate in the study. The experimental procedures were approved by the Ethical Committee

135 for the Psychological Research of the University of Padova by written consent (Ref. 2371) and

136 were in accordance with the Declaration of Helsinki.

138 Stimuli

139 Two video-clips showed an actress: i) pouring sugar with a tablespoon (grasped with a PG) in a 140 mug located nearby, and then stretching out her arm in an attempt to pour the sugar left in the 141 tablespoon within a mug located out of her reach (Interactive action; Fig. 2a), ii) pouring sugar in 142 the same mug, and then coming back to the starting point (Non-Interactive action; Fig. 2b).

143 Crucially, the out-of-reach mug was placed in the video foreground, closer to the participant 144 watching the video, thus eliciting a complementary reaction with a WHG when the actress was 145 trying to reach for it. The mug was visible in the video foreground also when the actress was 146 coming back to the starting point (Non-Interactive action), therefore controlling for possible 147 affordance effects. In order to assess that the joint action goal (i.e., trying to pour the sugar in the 148 mug) was clear to the participant, we performed a preliminary experiment. 50 participants (39 149 females and 11 males, age range 24-41) with the same characteristics of those participating in the 150 main experiment took part in this study. They were shown the interactive video-clip and they 151 were asked to indicate: i) whether they felt involved in the action and ii) what was the meaning 152 of the actress's gesture. We avoided any given option in order to elicit a more spontaneous 153 response. Results showed that 48 participants out of $50(96 \%)$ reported that they felt involved in 
154 the action and $46(92 \%)$ reported that the actress was 'asking' for the out-of-reach mug. Four

$155(8 \%)$ participants declared that the actress was 'indicating' the mug. When asked what they had

156 performed, they all (100\%) declared that they would have grabbed and lifted the mug.

157 All of the videos were taken from a frontal view and were equal in length (8.2 s). Since

158 gaze is a crucial component of social interactions and could have biased the results, the actress's

159 face was not visible. For the participants' prehension task we adopted a sugar spoon (130 mm

160 length, the same sugar spoon observed in the videos) vertically inserted into a mug (90 mm

161 diameter, the same mug observed in the videos). An affixed colored dot on the sugar spoon and

162 on the mug was signaling the required thumb's contact-point to perform stable and consistent

163 grasps across the experiment and across participants. Two auditory signals (a low-pitch tone, 300

$164 \mathrm{~Hz}, 200 \mathrm{~ms}$; and a high-pitch tone, $500 \mathrm{~Hz}, 200 \mathrm{~ms}$ ) were adopted as 'Go' signals at the

165 presentation of a white fixation cross, which lasted until the end of the trial (Fig. 3).

167 Procedure

168 The experimental set up is depicted in Figure 4. Participants sat on a chair in front of a table (900

$169 \times 900 \mathrm{~mm}$ ), watched the videos that were presented on a 19" monitor (resolution $1280 \times 1024$

170 pixels, refresh frequency $75 \mathrm{~Hz}$, background luminance of $0.5 \mathrm{~cd} / \mathrm{m}^{2}$ ) set at eye level (the eye-

171 screen distance was $60 \mathrm{~cm})$. A starting platform $(60 \times 70 \mathrm{~mm} ; 5 \mathrm{~mm}$ thick $)$ was attached $90 \mathrm{~mm}$

172 away from the table surface's edge and $50 \mathrm{~mm}$ away from the midsection. After video

173 presentation, participants had to execute a reach-to-grasp movement towards either a spoon or a

174 mug placed on a target platform $(100 \times 100 \mathrm{~mm} ; 5 \mathrm{~mm}$ thick $)$, located $350 \mathrm{~mm}$ from the starting

175 platform. The experiment included six experimental conditions; notably, the observed grasp was

176 always a PG:

Peer) reviewing PDF | (2019:04:37172:2:1:NEW 17 Aug 2019) 
177 - Interactive action, Performed PG (Interactive PG): participants performed a PG after observing

178 the Interactive request toward the mug.

179 - Interactive action, Performed WHG (Interactive WHG): participants performed a WHG after

180 observing the Interactive request toward the mug.

181 - Non-Interactive action, Performed PG (Non-Interactive PG): participants performed a PG after

182 observing the Non-Interactive action.

183 - Non-Interactive action, Performed WHG (Non-Interactive WHG): participants performed a

184 WHG after observing the Non-Interactive action.

185 - Baseline PG: participants performed a PG on the sugar spoon after observing a white cross

186 presented at the center of the monitor for $6 \mathrm{~s}$.

187 - Baseline WHG: participants performed a WHG on the mug after observing a white cross 188 presented at the center of the monitor for $6 \mathrm{~s}$.

189 The baseline conditions were performed before the experimental session to allow 190 participants to familiarize with the 'Go' signals and to provide baseline data for both types of 191 grasp. The experiment was composed of 60 trials (10 per condition, each lasting $11 \mathrm{~s})$. The 192 acoustic 'Go' signal was released at the offset of each video or at the offset of the fixation cross 193 in the baseline conditions. Participants were instructed to begin their movements as soon as the

194 'Go' signal sounded and to perform either a PG or a WHG. Trials were presented in randomized 195 order and the association between required type of grasp and corresponding auditory signal was 196 counterbalanced across participants. The time interval between the end of the video and the 197 presentation of the 'Go' signal was varied randomly to reduce rhythmical effects (1200-2400 ms 198 range). 
200

201

202

203

204

205

206

207

208

209

210

211

212

213

214

215

216

217

218

219

220

221

Kinematics recording

A 3D Optoelectronic SMART-D system (Bioengineering Technology and Systems, $\mathrm{B}|\mathrm{T}| \mathrm{S} \mid$ ) was used to track the kinematics of the participant's right upper limb. Six digital infrared cameras (sampling rate $60 \mathrm{~Hz}$ ) equipped with highly sensitive CCD sensors were placed in a semicircle at 1-1.2 meters from the table (Fig. 4a). The spatial resolution of the recording system was $0.3 \mathrm{~mm}$ over the field of view. Two reflective markers $(0.25 \mathrm{~mm}$ in diameter $)$ were placed on each participant's hand to measure the grasping component of the action (i.e., concerning finger preshaping and finger closing around the object), and one marker was placed on the wrist to measure the reaching component of the action (i.e., concerning hand transportation toward the target object). In particular, the three infrared reflective markers were taped to the following points: thumb (ulnar side of the nail), index finger (radial side of the nail), and wrist (dorsodistal aspect of the radial styloid process) (see Fig. 4b). Following data collection, the SMART-D Tracker software package $(\mathrm{B}|\mathrm{T}| \mathrm{S} \mid)$ was used to provide a 3D reconstruction of the markers' positions as a function of time.

\section{Data analysis}

The temporal delay between the 'Go' signal and movement onset (i.e., the time at which the tangential velocity of the wrist marker crossed a threshold of $5 \mathrm{~mm} / \mathrm{s}$ and remained above it for longer than $500 \mathrm{~ms}$ ) was computed as Reaction Time (RT). Movement Time (MT) was then computed as the time interval between reaching onset and end of grasping (i.e., the time at which the hand opening velocity crossed a threshold of $5 \mathrm{~mm} / \mathrm{s}$ after reaching its minimum value and remained above it for longer than $500 \mathrm{~ms}$ ). Further, the maximum distance reached by the 3D 
222 coordinates of the thumb and index finger (Maximum Grip Aperture, MGA) was extracted for

223 each individual movement.

224 The mean values for each parameter of interest were determined for each participant and

225 entered into repeated-measures 3x2 ANOVAs with Condition (Interactive, Non-Interactive,

226 Baseline) and Type of grasp (PG, WHG) as within-subject factors. Preliminary analyses were

227 conducted to check for normality, sphericity, univariate and multivariate outliers, with no

228 violations noted. Bonferroni correction was applied and a significance threshold level of $\mathrm{p}<0.05$

229 was set for all statistical analysis.

230

231 Results

232 A significant interaction of Condition by Type of Grasp was shown for $\operatorname{RTs}\left[\mathrm{F}_{(2,30)}=50.341, \mathrm{p}<\right.$

$\left.2330.001, \eta_{p}^{2}=0.770\right]$ and for $\operatorname{MT}\left[F_{(2,30)}=30.335, p<0.001, \eta^{2}=0.669\right]$. For MGA, a significant

234 effect of Type of Grasp $\left[\mathrm{F}_{(2,30)}=12.292, \mathrm{p}=0.003, \eta_{\mathrm{p}}^{2}=0.450\right]$ and a significant interaction of

235 Condition by Type of Grasp $\left[\mathrm{F}_{(2,30)}=5.872, \mathrm{p}=0.007, \eta_{\mathrm{p}}^{2}=0.281\right]$ emerged. The results

236 obtained from the post-hoc contrasts exploring the interactions are graphically represented in

237 Figure 5 and listed according to the main hypotheses:

238

239 Visuomotor priming

240 - RTs. Decreased RTs were found for the Non-Interactive PG compared to the Baseline PG

241 condition $\left(p=0.019, \eta_{p}^{2}=0.400\right.$; Fig. 5a).

242 - MT. Decreased MT was found for the Non-Interactive PG compared to the Baseline PG

243 condition $\left(p=0.015, \eta_{p}^{2}=0.419\right.$; Fig. 5b). 
244 - MGA. No significant effect was found for the Non-Interactive PG with respect to the Baseline

245 PG condition $\left(\mathrm{p}=1.00, \eta_{\mathrm{p}}^{2}=0.041\right.$; Fig. 5c).

246 Visuomotor interference

247 - RTs. Increased RTs were found for the Non-Interactive WHG compared to the Baseline WHG

248 condition $\left(p=0.008, \eta_{p}^{2}=0.466\right.$; Fig. 5d).

249 - MT. Increased MT was found for the Non-Interactive WHG compared to the Baseline WHG 250 condition $\left(p=0.038, \eta_{p}^{2}=0.348\right.$; Fig. 5e).

251 - MGA. No significant effect was found for the Non-Interactive WHG with respect to the

252 Baseline WHG condition $\left(p=0.781, \eta_{p}^{2}=0.084 ;\right.$ Fig. 5f).

253 Social motor priming

254 - RTs. Decreased RTs were found for the Interactive WHG compared to the Non-Interactive

255 WHG condition $\left(p<0.001, \eta_{p}^{2}=0.780\right.$; Fig. 5d) and to Baseline WHG values $\left(p=0.002, \eta_{p}^{2}=\right.$ 256 0.537; Fig. 5d).

257 - MT. Decreased MT was found for the Interactive WHG compared to the Non-Interactive WHG 258 condition $\left(p=0.005, \eta_{p}^{2}=0.499\right)$ and to Baseline WHG values $\left(p=0.036, \eta_{p}^{2}=0.353 ;\right.$ Fig. 5e).

259 - MGA. No significant effect was found for the Interactive WHG with respect to Non-Interactive 260 WHG $\left(p=1.00, \eta_{p}^{2}=0.005\right)$ and to Baseline WHG values $\left(p=0.633, \eta^{2}=0.102 ;\right.$ Fig. 5f $)$.

262 Social motor interference

263 - RTs. Increased RTs were found for the Interactive PG compared to the Non-Interactive PG

264 condition $\left(p<0.001, \eta_{p}^{2}=0.689\right)$ and to Baseline PG values $\left(p=0.021, \eta_{p}^{2}=0.393\right.$; Fig. 5a).

265 - MT. Increased MT was found for the Interactive PG compared to the Non-Interactive PG

266 condition $\left(p=0.001, \eta_{p}^{2}=0.584\right)$ and to Baseline PG values $\left(p=0.021, \eta_{p}^{2}=0.395\right.$; Fig. 5b). 
267 - MGA. A significant effect was found for MGA. In particular, grip aperture was increased when

268 performing a PG for the Interactive condition compared to the Non-Interactive condition $(\mathrm{p}=$

$\left.2690.038, \eta_{p}^{2}=0.349\right)$ and to Baseline PG values $\left(p=0.008, \eta_{p}^{2}=0.463 ;\right.$ Fig. 5c).

270

\section{Discussion}

272 This study aimed to investigate social motor priming effects in a postponed task (i.e., offline).

273 Our findings confirmed earlier research by showing that action observation facilitates congruent

274 types of actions (visuomotor priming) and interferes with different types of actions (visuomotor

275 interference). At the same time, our results extend previous literature on social interactions by

276 showing that interactive requests can facilitate incongruent - but appropriate - responses (i.e.,

277 social motor priming) and interfere with congruent - but inappropriate - grasping actions (i.e.,

278 social motor interference). Notably, this effect spontaneously occurs even in a postponed task.

Social vs. non-social motor interference

281 Do visuomotor and social motor interference involve overlapping or distinct mechanisms?

282 Interestingly, we found a crucial dissociation. Social motor interference inflicted a more serious

283 cost on kinematics than visuomotor interference. MGA was significantly increased when

284 participants performed a PG after having (spontaneously) planned a WHG. No modulation of the

285 grasping component was found in the case of visuomotor interference. Given that grip amplitude

286 covaries linearly with object size (Jakobson \& Goodale, 1991), the change in MGA was

287 seemingly due to the previously observed social request. This output (i.e., an increased hand

288 aperture) possibly indicates the integration of another motor plan (i.e., a WHG) when performing

289 the offline precision grip. The present data take previous results a step further by demonstrating 
290 that social motor priming - once spontaneously triggered - is rather impervious to be inhibited

291 even during postponed action execution. In fact, it affects both the reaching and the grasping

292 components of performed actions. This might ultimately suggest that social motor priming is

293 more pervasive than visuomotor priming. Notably, shifting from imitating other's action to

294 representing the most appropriate complementary response occurs very quickly (i.e., functional

295 shift; Sartori et al., 2013). Here we suggest instead that shifting from preparing a complementary

296 response to mirroring is very expensive in terms of motor interference. Is this due to the

297 intrinsically social valence of the stimulus? In this regard, we recently provided evidence that

298 activity in the primary motor cortex elicited by a complementary request is even impermeable to

299 attention-diverting cues (Betti et al., 2017). Social motor preparation seems therefore to be a

300 genuine automatic (i.e., not reprogrammable) mechanism.

301

302 Integration as a byproduct of offline social motor interference

303 Previous reports on simultaneous observation-execution interference tasks (i.e., online

304 interference; Chinellato, Castiello \& Sartori, 2015) indicated that planning a socially-appropriate

305 WHG had a repulsive effect on what was performed (i.e., decreasing the MGA of the PG). This

306 effect was likely driven by a form of inhibition of the features shared by perception and action

307 (for a review see Castiello, 1999). According to Schubö and colleagues (2001), the

308 representations that underlie different activities, such as producing a movement while

309 simultaneously coding a perceptual event, must be kept distinct so that the two activities can be

310 carried out without interfering (Schubö, Aschersleben \& Prinz, 2001). Rather, our results for the

311 postponed task (i.e., offline interference) suggest an integration of the inhibited motor plan (i.e.,

312 a WHG) into the executed action. 
313 In future studies, the adoption of real-life meaningful interactions involving a set of different

314 potential actions (e.g., Becchio et al., 2008) will permit to ascertain whether the integration

315 documented here also generalize to more complex contexts and situations. For instance, dynamic

316 interactions will allow testing pair's mutual compensation when the poor performance of one

317 individual is fixed at the dyadic level (Era et al., 2018).

The Continuum Hypothesis

320 In terms of online interference, participants' motor performance is less affected when they have

321 to interact in a joint task with respect to an isolated context (Sacheli et al., 2018). In other

322 circumstances, however, the interference due to physical incongruence is only partially

323 decreased (Clarke et al., 2018) - or even increased (della Gatta et al. 2017) - by the joint goal.

324 For instance, when participants have to draw circles or lines with their right hands while

325 observing on the screen the circles or lines that are simultaneously drawn by a partner, this leads

326 to an increase - rather than a decrease - in interference effects during the Joint Action condition.

327 Based on these findings, we hypothesize that if a joint action is well consolidated in memory,

328 then less resources are needed to process it, and therefore less interference occurs. Likewise, less

329 interference occurs for a "second task" if the primary task is automatized (Castiello \& Umiltà,

330 1987; Castiello, 1996; Guillery, Mouraux \& Thonnard, 2013). Indeed, while the task in Sacheli

331 and colleagues' study (2018) was well learnt after a training phase of 20 minutes, Clarke and

332 colleagues' (2018) participants performed only 4 trials before each block, and no training at all

333 was performed by della Gatta and colleagues (2017). When a particular stimulus-response pair is

334 well learnt, a reduction in top-down control would indicate its automaticity over other less

335 frequently experienced responses (Campbell \& Cunnington, 2017). Altogether, these findings 
336 raise the possibility that motor interference is an experience-based continuum, rather than an

337 on/off mechanism. This hypothesis would also explain why the life-long learnt response of hand

338 shacking is so automatic (Liepelt et al., 2010). On the contrary, one might argue that interference

339 is a simple on/off effect produced by the online competition between different simultaneous

340 representations. In that case, motor interference should vanish in a postponed task since

341 competition would be no longer active. But in the present experiment this was not the case. Our

342 findings extend previous research by revealing that physically incongruent action representations

343 can be integrated into a single action plan even during an offline task and without any training.

344 Notably, our paradigm did not entail any imitative or complementary blocks and no instructions

345 regarding the action to be performed were given before trials began, as it occurred in other

346 previous studies (Newman-Norlund et al., 2007; van Schie, van Waterschoot \& Bekkering, 2008;

347 Poljac, Schie \& Bekkering, 2009; Ocampo \& Kritikos, 2010; Ocampo, Painter \& Kritikos,

348 2012). This approach was adopted in order to investigate a purely spontaneous phenomenon,

349 rather than a learnt effect. This aspect is important as in real life many cooperative and

350 competitive contexts require partners to flexibly adapt their responses to others' actions, without

351 the chance to previously know or even practice the specific response that they must implement

352 (e.g., Becchio et al., 2008; Sacheli et al., 2015).

353

354 Social response: Low-level or high-level mapping?

355 According to the Social Associative Memory hypothesis, an associative mechanism would be in

356 charge of matching certain actions to their natural social response, irrespective of who is actually

357 performing the action (Chinellato et al., 2013; Chinellato, Castiello \& Sartori, 2015). "If action B

358 (e.g., take) usually follows action A (e.g., give), the observation of a partner executing A elicits 
359 the pre-planning of B by the observer. On the other hand, if the subject executes A, he expects to

360 see the partner performing B in response" (Chinellato et al., 2013). Here, extensive experience of

361 carrying out complementary actions in a social context would result in automatically generating

362 the complementary action when observing an action in a social context. Consistent literature on

363 social Simon effects (Guagnano, Rusconi \& Umiltà, 2010; Humphreys \& Bedford, 2011;

364 Dittrich et al., 2013) and the development of Stimulus-Response associations (Catmur, Walsh \&

365 Heyes, 2009) provides convergent data on the hypothesis of a low-level direct mechanism for the

366 priming of different behaviors. Our results, based on a postponed interference effect, might

367 indeed be attributable to general processes of associative learning (Catmur, Walsh \& Heyes,

368 2009; Massen \& Prinz, 2009).

369 An alternative account for the present data is the high-level mapping. The theory of event

370 coding (TEC; Hommel \& Elsner, 2009; Hommel, 2009) states that observed (and executed)

371 actions are represented in the form of their distal consequences. The TEC is based on the

372 common coding hypothesis, which claims that perception and action rely on shared cognitive

373 representations. According to the TEC, translating a perceived human movement into

374 corresponding motor programs would function as an emulator, tracking the behavior of

375 conspecifics in real time to generate predictions of an unfolding action (Wilson \& Knoblich,

376 2005). Our data, on the contrary, show that observing others' behaviors rapidly activates

377 appropriate complementary motor plans in an observer. In fact, since the distal goal of the actor

378 is to reach the distant cup and the most efficient action to do it (by herself) would be slightly

379 rising from her seat, motor prediction should have activated in the observer the corresponding

380 leg muscles, rather than right-hand muscles. On the other hand, it is plausible that both a

381 predictive and a social motor response preparation might have taken place, as we recently 
382 demonstrated (Sartori et al., 2015). In conclusion, the reported effects are an example of a

383 spontaneous tendency to fulfill the request embedded in a social interaction. This might be

384 confirmed by the fact that a control group reported that they were ready to lift the salient object

385 toward the model. It could be argued that attention played a role in modulating motor priming

386 and that the actor's hand - moving toward the object - was simply more salient then the hand

387 moving back to the starting position, without the effect being intrinsically a social motor

388 priming. If this were the case, then a simple arrow presented instead of the hand would have

389 produced similar findings. However, results from previous studies in which the social request

390 was substituted by an arrow did not provide support for this view (Flach et al., 2010; Sartori et

391 al., 2011). Rather we suggest that the motor system is preferentially tuned to meaningful actions

392 of interactive partners.

393

394 Conclusions

395 The purpose of this study was to investigate whether visuomotor interference can modulate 396 perception-action coupling even after action observation (i.e., offline interference). The present

397 results suggest that physically incongruent action representations can be integrated into a single 398 action plan even during an offline task and without any training. The future goal is to design

399 stringent paradigms that might allow to compare findings from real-life meaningful interactions 400 involving a set of different potential actions. 
401 References

402 Bardi L, Bundt C, Notebaert W, Brass M. 2015. Eliminating mirror responses by instructions.

403

404

405

406

407

408

409

410

411

412

413

414

415

416

417

418

419

420

421

422

423

Cortex 70:128-136. DOI: 10.1016/j.cortex.2015.04.018

Becchio C, Sartori L, Bulgheroni M, Castiello U. 2008. Both your intention and mine are reflected in the kinematics of my reach-to-grasp movement. Cognition 106:894-912. DOI: 10.1016/j.cognition.2007.05.004

Betti S, Castiello U, Guerra S, Sartori L. 2017. Overt orienting of spatial attention and corticospinal excitability during action observation are unrelated. PLOS ONE 12:e0173114. DOI: 10.1371/journal.pone.0173114.

Brass M, Bekkering H, Prinz W. 2001. Movement observation affects movement execution in a simple response task. Acta Psychologica 106:3-22. DOI: 10.1016/S0001-6918(00)00024$\mathrm{X}$.

Brass M, Bekkering H, Wohlschläger A, Prinz W. 2000. Compatibility between Observed and Executed Finger Movements: Comparing Symbolic, Spatial, and Imitative Cues. Brain and Cognition 44:124-143. DOI: 10.1006/brcg.2000.1225.

Brass M, Derrfuss J, von Cramon DY. 2005. The inhibition of imitative and overlearned responses: a functional double dissociation. Neuropsychologia 43:89-98. DOI: 10.1016/j.neuropsychologia.2004.06.018.

Campbell MEJ, Cunnington R. 2017. More than an imitation game: Top-down modulation of the human mirror system. Neuroscience \& Biobehavioral Reviews 75:195-202. DOI: 10.1016/j.neubiorev.2017.01.035

Castiello U. 1996. Grasping a fruit: Selection for action. Journal of Experimental Psychology: Human Perception and Performance 22:582-603. DOI: 10.1037/0096-1523.22.3.582. 
424 Castiello U. 1999. Mechanisms of selection for the control of hand action. Trends in Cognitive 425 Sciences 3:264-271. DOI: 10.1016/S1364-6613(99)01346-7.

426 Castiello U, Umiltà C. 1987. Spatial compatibility effects in different sports. International $427 \quad$ Journal of Sport Psychology 18:276-285.

428 Catmur C, Gillmeister H, Bird G, Liepelt R, Brass M, Heyes C. 2008. Through the looking glass: counter-mirror activation following incompatible sensorimotor learning. European Journal of Neuroscience 28:1208-1215. DOI: 10.1111/j.1460-9568.2008.06419.x.

431

432

433

434

435

436

437

438

439

440

441

442

443

444

445

446

Catmur C, Walsh V, Heyes C. 2009. Associative sequence learning: the role of experience in the development of imitation and the mirror system. Philosophical Transactions of the Royal Society of London. Series B, Biological Sciences 364:2369-2380. DOI: 10.1098/rstb.2009.0048.

Chinellato E, Castiello U, Sartori L. 2015. Motor interference in interactive contexts. Frontiers in Psychology 6. DOI: 10.3389/fpsyg.2015.00791.

Chinellato E, Ognibene D, Sartori L, Demiris Y. 2013. Time to Change: Deciding When to Switch Action Plans during a Social Interaction. In: Lepora NF, Mura A, Krapp HG, Verschure PFMJ, Prescott TJ eds. Biomimetic and Biohybrid Systems. Berlin, Heidelberg: Springer Berlin Heidelberg, 47-58. DOI: 10.1007/978-3-642-39802-5_5.

Clarke S, Francová A, Székely M, Butterfill S, Michael J. 2018. Can Joint Action Goals Reduce Visuomotor Interference Effects from a Partner's Incongruent Actions?. PsyArXiv. DOI: 10.31234/osf.io/tdbne.

Cook R, Press C, Dickinson A, Heyes C. 2010. Acquisition of automatic imitation is sensitive to sensorimotor contingency. Journal of Experimental Psychology: Human Perception and Performance 36:840-852. DOI: 10.1037/a0019256. 
447 Cross KA, Iacoboni M. 2014a. Neural systems for preparatory control of imitation.

448

449

450

451

452

453

454

455

456

457

458

459

460

461

462

463

464

465

466

467

468

469

Philosophical Transactions of the Royal Society B: Biological Sciences, 369:20130176.

DOI: $10.1098 /$ rstb.2013.0176

Cross KA, Iacoboni M. 2014b. To imitate or not: Avoiding imitation involves preparatory inhibition of motor resonance. NeuroImage 91:228-236. DOI:

10.1016/j.neuroimage.2014.01.027.

della Gatta F, Garbarini F, Rabuffetti M, Viganò L, Butterfill SA, Sinigaglia C. 2017. Drawn together: When motor representations ground joint actions. Cognition 165:53-60. DOI: 10.1016/j.cognition.2017.04.008

Dittrich K, Dolk T, Rothe-Wulf A, Klauer KC, Prinz W. 2013. Keys and seats: Spatial response coding underlying the joint spatial compatibility effect. Attention, Perception, \& Psychophysics 75:1725-1736. DOI: 10.3758/s13414-013-0524-z.

Edwards MG, Humphreys GW, Castiello U. 2003. Motor facilitation following action observation: A behavioural study in prehensile action. Brain and Cognition 53:495-502. DOI: $10.1016 / \mathrm{S} 0278-2626(03) 00210-0$.

Era V, Aglioti SM, Mancusi C, Candidi M. 2018. Visuo-motor interference with a virtual partner is equally present in cooperative and competitive interactions. Psychological Research:113. DOI: $10.1007 / \mathrm{s} 00426-018-1090-8$

Era V, Candidi M, Gandolfo M, Sacheli LM, Aglioti SM. 2018. Inhibition of left anterior intraparietal sulcus shows that mutual adjustment marks dyadic joint-actions in humans. Social Cognitive and Affective Neuroscience 13:492-500. DOI: 10.1093/scan/nsy022

Fitts PM, Seeger CM. 1953. S-R compatibility: spatial characteristics of stimulus and response codes. Journal of Experimental Psychology 46:199-210. DOI: 10.1037/h0062827

Peer) reviewing PDF | (2019:04:37172:2:1:NEW 17 Aug 2019) 
470 Flach R, Press C, Badets A, Heyes C. 2010. Shaking hands: Priming by social action effects.

471

472

473

474

475

476

477

478

479

480

481

482

483

484

485

486

487

488

489

490

491 British Journal of Psychology 101:739-749. DOI: 10.1348/000712609X484595.

Gandolfo M, Era V, Tieri G, Sacheli LM, Candidi M. 2019. Interactor's body shape does not affect visuo-motor interference effects during motor coordination. Acta Psychologica 196:42-50. DOI: 10.1016/j.actpsy.2019.04.003

Gowen E, Poliakoff E. 2012. How does visuomotor priming differ for biological and nonbiological stimuli? A review of the evidence. Psychological Research 76:407-420. DOI: 10.1007/s00426-011-0389-5.

Guagnano D, Rusconi E, Umiltà CA. 2010. Sharing a task or sharing space? On the effect of the confederate in action coding in a detection task. Cognition 114:348-355. DOI: 10.1016/j.cognition.2009.10.008.

Guillery E, Mouraux A, Thonnard J-L. 2013. Cognitive-Motor Interference While Grasping, Lifting and Holding Objects. PLOS ONE 8:e80125. DOI: 10.1371/journal.pone.0080125.

Hamilton AF de C. 2013. The mirror neuron system contributes to social responding. Cortex 49:2957-2959. DOI: 10.1016/j.cortex.2013.08.012.

Heyes C. 2011. Automatic imitation. Psychological Bulletin 137:463-483. DOI: $10.1037 / \mathrm{a} 0022288$.

Hommel B. 2009. Action control according to TEC (theory of event coding). Psychological Research Psychologische Forschung 73:512-526. DOI: 10.1007/s00426-009-0234-2.

Hommel B, Elsner B. 2009. Acquisition, representation, and control of action. In: Oxford handbook of human action. Social cognition and social neuroscience. New York, NY, US: Oxford University Press, 368-397. 
492 Humphreys GW, Bedford J. 2011. The relations between joint action and theory of mind: a

493

494

495

496

497

498

499

500

501

502

503

504

505

506

507

508

509

510

511

512

513

514 neuropsychological analysis. Experimental Brain Research 211:357-369. DOI: 10.1007/s00221-011-2643-X.

Jakobson LS, Goodale MA. 1991. Factors affecting higher-order movement planning: a kinematic analysis of human prehension. Experimental Brain Research 86. DOI: 10.1007/BF00231054.

Kilner JM, Paulignan Y, Blakemore SJ. 2003. An Interference Effect of Observed Biological Movement on Action. Current Biology 13:522-525. DOI: 10.1016/S09609822(03)00165-9

Krishnan-Barman S, Forbes PAG, Hamilton AF de C. 2017. How can the study of action kinematics inform our understanding of human social interaction? Neuropsychologia 105:101-110. DOI: 10.1016/j.neuropsychologia.2017.01.018.

Leighton J, Bird G, Orsini C, Heyes C. 2010. Social attitudes modulate automatic imitation. Journal of Experimental Social Psychology 46:905-910. DOI: 10.1016/j.jesp.2010.07.001

Liepelt R, Prinz W, Brass M. 2010. When do we simulate non-human agents? Dissociating communicative and non-communicative actions. Cognition 115:426-434. DOI: 10.1016/j.cognition.2010.03.003.

Massen C, Prinz W. 2009. Movements, actions and tool-use actions: an ideomotor approach to imitation. Philosophical Transactions of the Royal Society B: Biological Sciences 364:2349-2358. DOI: 10.1098/rstb.2009.0059.

Newman-Norlund RD, van Schie HT, van Zuijlen AMJ, Bekkering H. 2007. The mirror neuron system is more active during complementary compared with imitative action. Nature Neuroscience 10:817-818. DOI: 10.1038/nn1911. 
515 Ocampo B, Kritikos A. 2010. Placing actions in context: motor facilitation following observation

516 of identical and non-identical manual acts. Experimental Brain Research 201:743-751.

517 DOI: $10.1007 / \mathrm{s} 00221-009-2089-6$.

518 Ocampo B, Painter DR, Kritikos A. 2012. Event coding and motor priming: how attentional 519 modulation may influence binding across action properties. Experimental Brain Research 219:139-150. DOI: 10.1007/s00221-012-3073-0.

Poljac E, Schie HT, Bekkering H. 2009. Understanding the flexibility of action-perception coupling. Psychological Research Psychologische Forschung 73:578-586. DOI: 10.1007/s00426-009-0238-y.

Sacheli LM, Arcangeli E, Paulesu E. 2018. Evidence for a dyadic motor plan in joint action. Scientific Reports 8:5027. DOI: 10.1038/s41598-018-23275-9. action with an out-group avatar. Scientific Reports 5:8507. DOI: 10.1038/srep08507

Sartori L. 2016. Complementary Actions. In Obhi \& Cross (Eds.) Shared Representations: Sensorimotor Foundations of Social Life (pp. 392-416). Cambridge University Press.

531 Sartori L, Betti S. 2015. Complementary actions. Frontiers in Psychology 6. DOI: 10.3389/fpsyg.2015.00557.

533 Sartori L, Betti S, Chinellato E, Castiello U. 2015. The multiform motor cortical output:

534 Kinematic, predictive and response coding. Cortex 70:169-178. DOI: 10.1016/j.cortex.2015.01.019.

536 Sartori L, Bucchioni G, Castiello U. 2013. When emulation becomes reciprocity. Social Cognitive and Affective Neuroscience 8:662-669. DOI: 10.1093/scan/nss044. 
538 Sartori L, Cavallo A, Bucchioni G, Castiello U. 2011. Corticospinal excitability is specifically

539 modulated by the social dimension of observed actions. Experimental Brain Research 211:557. DOI: 10.1007/s00221-011-2650-y.

541 Schubö A, Aschersleben G, Prinz W. 2001. Interactions between perception and action in a 542 reaction task with overlapping S-R assignments. Psychological Research 65:145-157.

$543 \quad$ DOI: $10.1007 / \mathrm{s} 004260100061$.

544 Scorolli C, Miatton M, Wheaton LA, Borghiac AM. I give you a cup, I get a cup: A kinematic $545 \quad$ study on social intention. Neuropsychologia 57:196-204. DOI:

$546 \quad$ 10.1016/j.neuropsychologia.2014.03.006

547 Sparks S, Douglas T, Kritikos A. 2016. Verbal social primes alter motor contagion during action 548 observation. The Quarterly Journal of Experimental Psychology 69:1041-1048. DOI: $10.1080 / 17470218.2015 .1113304$

van Schie HT, van Waterschoot BM, Bekkering H. 2008. Understanding action beyond 551 34:1493-1500. DOI: 10.1037/a0011750.

Wang Y, Hamilton AF de C. 2013. Understanding the Role of the "Self" in the Social Priming of Mimicry. PLoS ONE 8:e60249. DOI: 10.1371/journal.pone.0060249.

Wilson M, Knoblich G. 2005. The case for motor involvement in perceiving conspecifics. Psychological Bulletin 131:460-473. DOI: 10.1037/0033-2909.131.3.460. 


\section{Figure 1}

\section{Experimental Hypotheses}

a) Visuomotor priming is expected when the performed grip (PG) is matched with the observed non-interactive action. b) Visuomotor interference is expected when the performed grip (WHG) is mismatched with the observed non-interactive action. c) Social motor priming is expected when the performed grip (WHG) is matched with the social request directed to the mug. d) Social motor interference is expected when the performed grip (PG) is mismatched with the social request directed to the mug. 
NON-INTERACTIVE

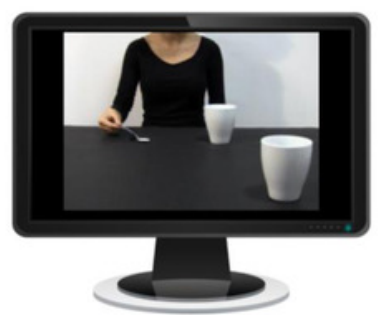

OBSERVED GRIP:

$P G$

a

Performed GRIP

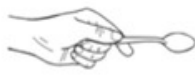

PG

b

PERformed GRIP

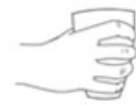

WHG
Visuomotor priming
INTERACTIVE

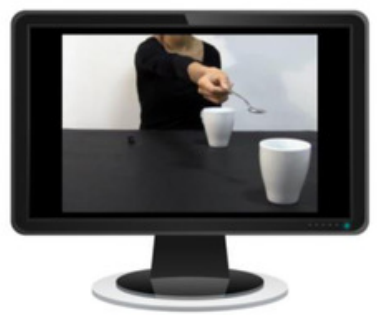

OBSERVED GRIP:

PG

d

Social motor interference
C

Visuomotor interference
Social motor priming 


\section{Figure 2}

Experimental stimuli

a) Interactive action: The actress pours sugar with a tablespoon (precision grip; PG) in a mug located nearby, and then stretches out her arm trying to pour some sugar into a mug located out of her reach (red circle). Crucially, this mug is placed in the video foreground, thus requiring the observer's intervention to bring the mug closer. b) Non-Interactive action: The actress pours sugar in the same mug, and then comes back to the starting point.

a

INTERACTIVE
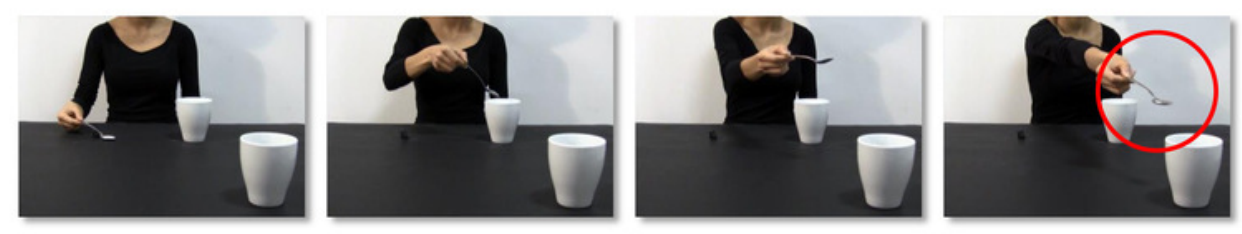

b

NON-INTERACTIVE
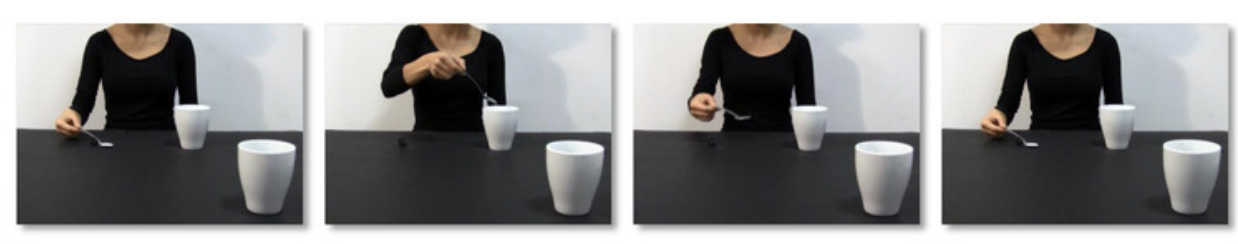

Timeline 


\section{Figure 3}

Timeline

During the Interactive condition, the participants observed a video showing an actress stretching out her arm trying to pour some sugar into a mug located close to them, thereby inviting them to grasp it. Depending on the specific type of ' $G o$ ' signal, they then performed a reach-to-grasp task on the mug or on the spoon located on a target platform.

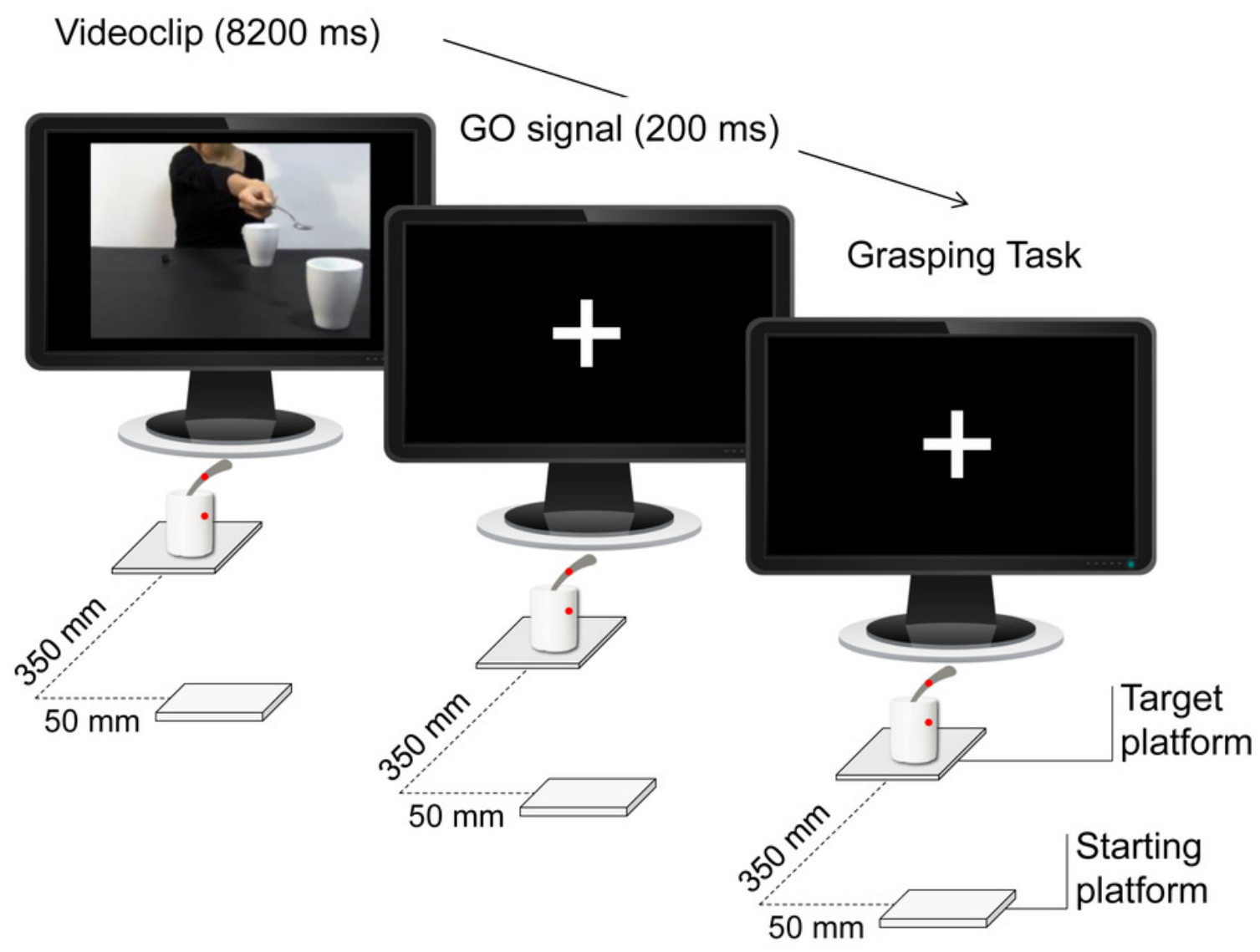




\section{Figure 4}

Set up

a) A 3D Optoelectronic SMART-D system was used to track the kinematics of the participant's right upper limb by means of six infrared cameras equipped with highly sensitive CCD sensors. Each participant sat in front of a table and had to watch the video clips (Interactive, Non-Interactive) that were presented on a monitor. b) The participant's right elbow and wrist were resting on the table surface with the hand resting on a starting platform. To measure the grasp and reach components of the movement, retro reflecting markers were taped to the following points: thumb, index finger, and wrist. 


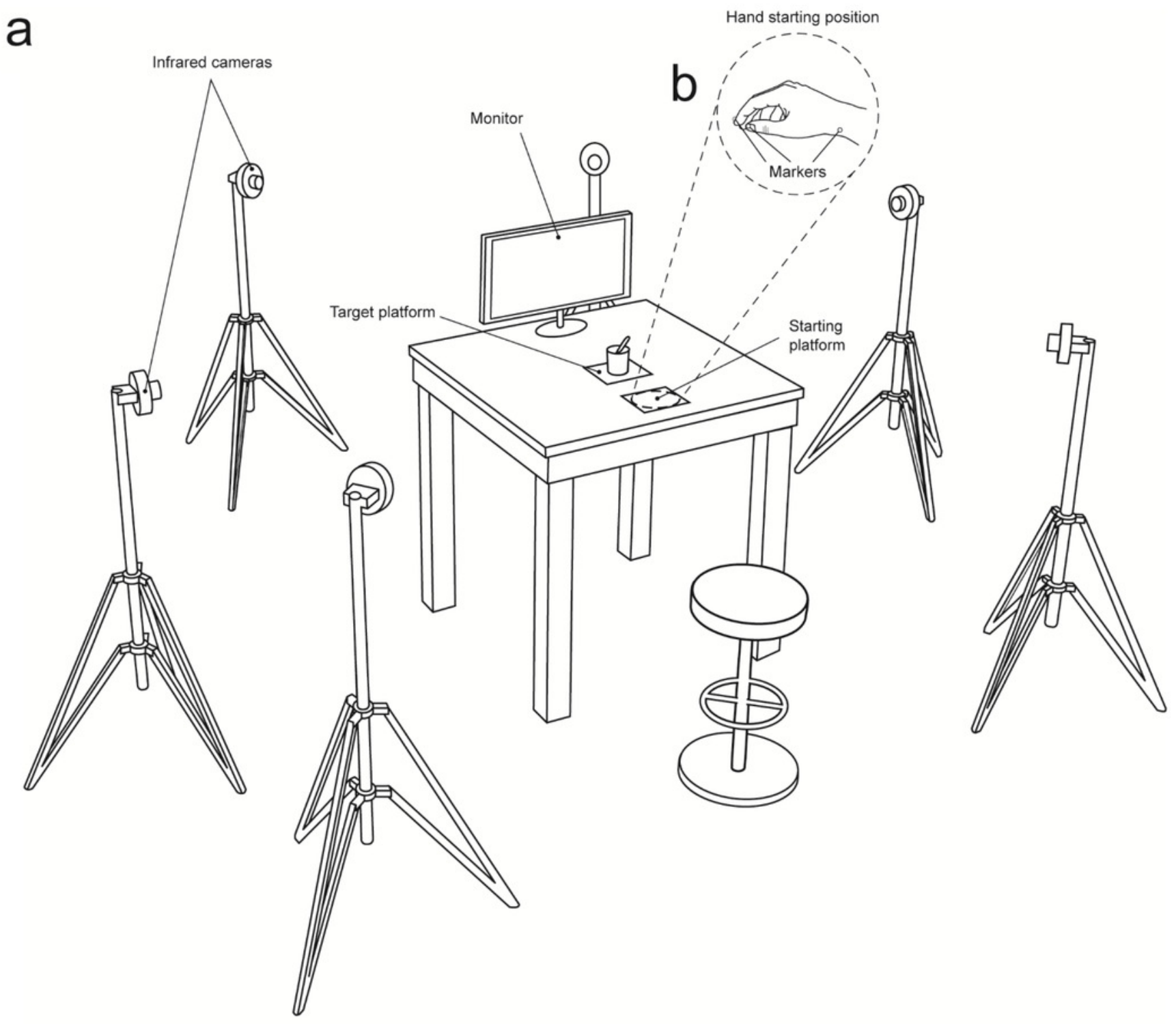




\section{Figure 5}

Results

Graphical representation of the mean values for RTs (black; a,d), MT (gray; b,e) and MGA (white; c,f) across experimental conditions (Interactive, Non-Interactive, Baseline) when participants either performed a PG (left column) or a WHG (right column). Bars represent standard error of the mean. Asterisks indicate statistically significant comparisons, $(*) p<$ 0.05, $(* *) p<0.01$. 


\section{PRECISION GRIP}
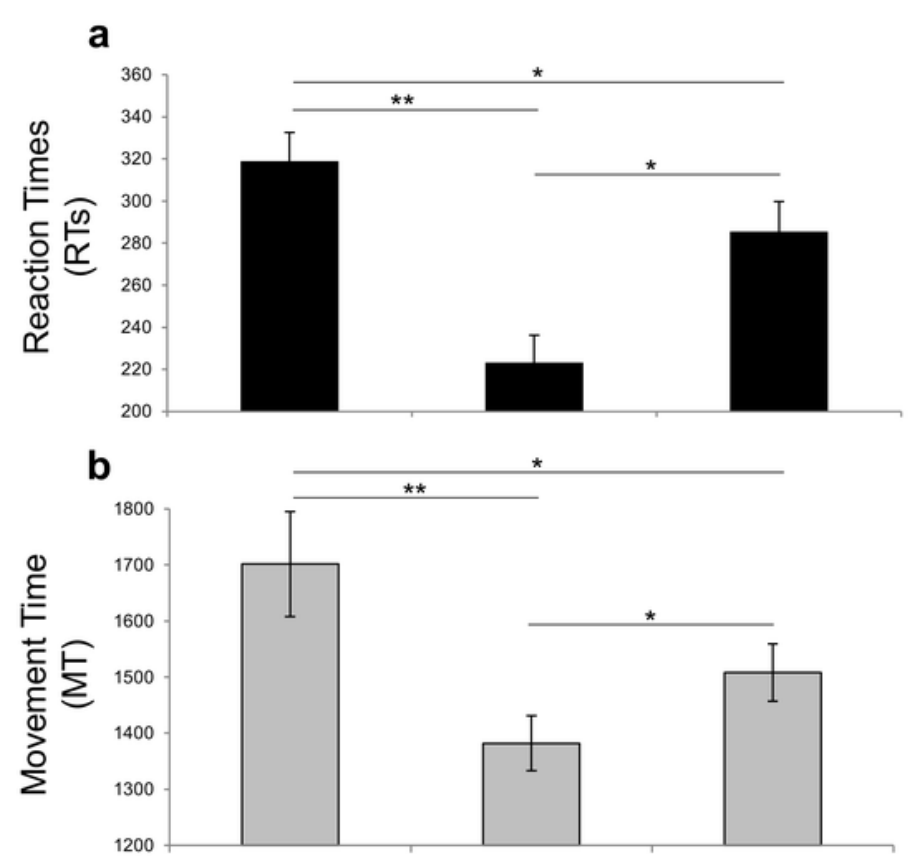

C

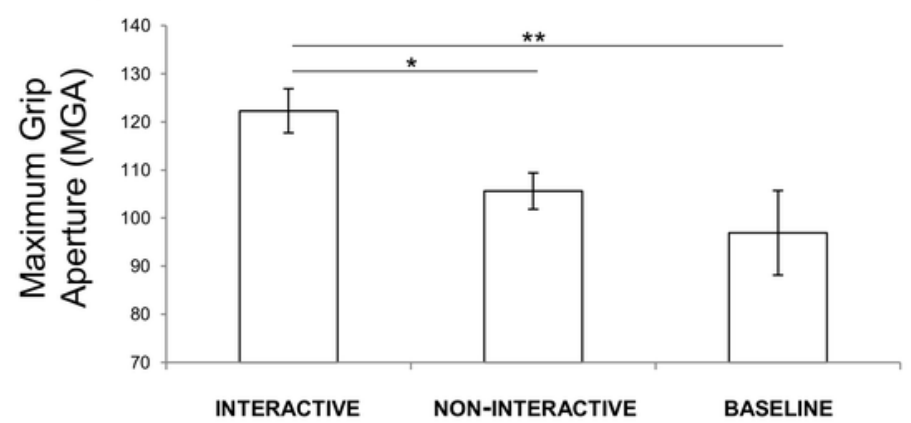

d

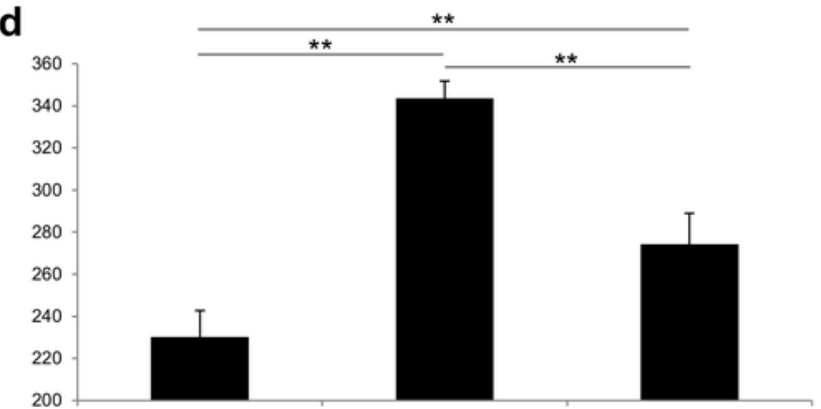

e

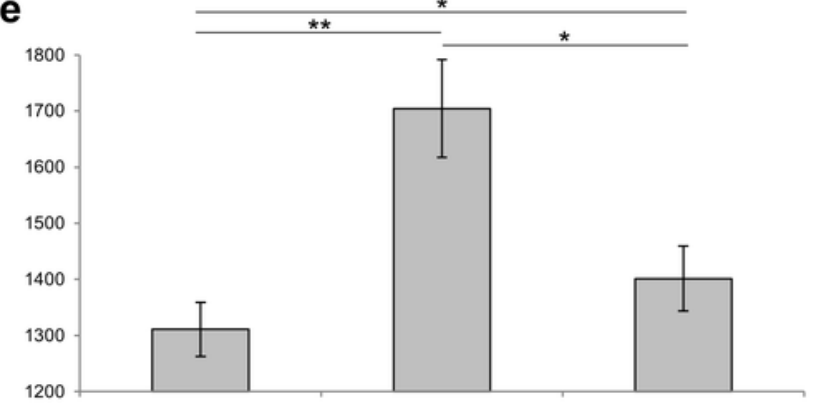

f

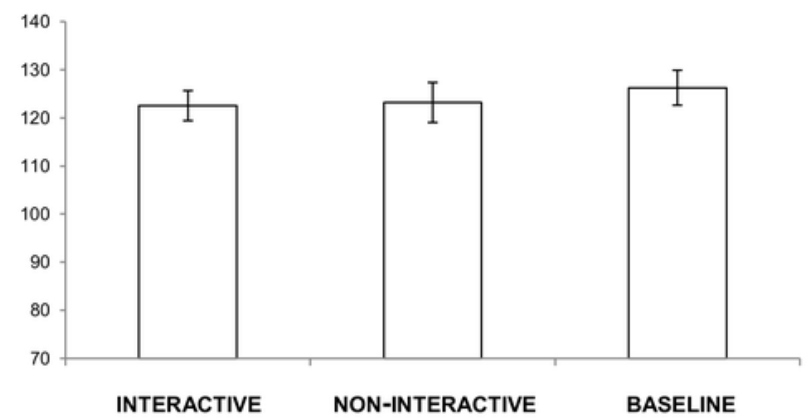

